\title{
Le Noir au théâtre: de la violence à l'écosophie
}

\author{
O Escuro no Teatro: \\ da violência à ecosofia
}

\section{Darkness in the Theatre: From Violence to Ecosophy}

Véronique Perruchon 


\section{Résumé}

Cet article porte sur la dimension profonde du noir au théâtre. Ce travail propose de répondre à la question sousjacente au thème : comment parler de l'obscurité dans sa dimension la plus profonde? Pour ce faire, le texte s'appuie sur des spectacles créés en France et de renommée internationale.

Mots-clés: Noir au théâtre ; lumière du spectacle ; créateurs lumière

\section{Resumo}

Este artigo aborda a dimensão mais profunda do escuro em cena. $O$ trabalho se propõe a responder à questão subjacente ao tema, qual seja, como falar do escuro em sua dimensão mais profunda? Para tanto a sua escrita se pauta em espetáculos de renome internacional criados na França.

Palavras-chaves: Escuro da cena; luz do espetáculo; criadores de iluminação

\section{Abstract}

This article deals with the deeper dimension of darkness on stage. The text proposes to answer the underlying question: how to talk about darkness in the theatre, in its deepest dimension? In order to do so, this article is based on performances of international reputation created in France.

Keywords: Darkness in the theatre; stage lighting; lighting designers

E-ISSN: 2358.6958

\footnotetext{
${ }^{1}$ Véronique Perruchon, Professeure en Arts de la Scène à l'Université de Lille et membre du Centre d'Études des Arts contemporains, dirige un programme de recherche sur la "Lumière de Spectacle ». Après des débuts professionnels en tant qu'éclairagiste et régisseuse, elle s'est intéressée au domaine spectaculaire du théâtre et à la mise en scène dans son travail de recherche. Elle publie aux Presses universitaires du Septentrion, les ouvrages : Noir. Lumière et théâtralité en 2016, et André Engel CEuvre théâtrale en 2018. Références intitutionnelles: LdS Programme de recherche "Lumière de Spectacle ». Université de Lille. EA 3587 - CEAC - Centre d'Étude des Arts Contemporains. 59000 Lille, France.
} 
Pour ce numéro de la revue Urdimento consacré à la lumière, il me paraît important de parler du noir en l'abordant dans sa dimension la plus profonde et par là même de nous confronter à ce qu'il a de plus dérangeant ou envoûtant. Cependant, je ne voudrais pas nous détourner de la lumière de spectacle en glosant sur les dimensions philosophiques et psychologiques du noir à travers des interprétations symboliques. L'aborder selon cette méthode nous mènerait directement à l'intellection. Or, comme il ne suffit pas de penser pour être, selon la proclamation de Descartes, mais de vivre, vivons l'expérience du noir pour mieux le penser. Aussi je me propose de répondre à la question sous-jacente du sujet - comment parler du noir dans sa dimension la plus profonde ? - à partir de l'expérience. Celle des performeurs (acteurs, danseurs, interprètes, etc.), celle des spectateurs, et celle des créateurs (chorégraphes, metteurs en scène, éclairagistes). Je ferai référence pour cela à des spectacles souvent créés en France mais ayant une renommée internationale ${ }^{2}$. Nous verrons à travers ces considérations comment le noir brut peut se laisser apprivoiser et générer des atmosphères, des émotions et des pensées divergentes.

\title{
De la toute-puissance lumineuse...
}

La lumière de spectacle a suivi historiquement et contextuellement une course à la puissance. De la conquête de la lumière sur le noir aux XVle et XVIle siècles - avec comme point de repère les fêtes à Versailles en 1664 et 1668 du bien nommé Roi Soleil qui offrit un éblouissement réel et symbolique à ses courtisans, jusqu'aux multiplications de matériel pour éclairer un spectacle depuis l'électrification des théâtres au XXe siècle, l'histoire de la lumière nous montre cette quête ${ }^{3}$. Je ne citerai ici que le texte de Louis Jouvet qui décrivait en 1937 cette montée en puissance des lumières de spectacle de manière comparative :

\begin{abstract}
Si on envisage l'instrument d'éclairage qu'est la rampe, nous pouvons, d'après les gravures de l'époque, dénombrer à peu près ses éléments : une rampe de trente chandelles devait être déjà un luxe ; la bougie ne donne point un grand progrès sur la chandelle ( 9 bougies équivalent à 10 chandelles); mais le quinquet vaut déjà 6 bougies de cire ; la lampe Carcel qui lui succède (et qui servira plus tard d'étalon pour déterminer la bougie décimale) a une puissance égale à 9 bougies décimales; puis vient le bec papillon qui donne 15 bougies, et enfin le bec Auer, 120 bougies. [...]. Aujourd'hui, la rampe de la Comédie Française comporte quarante-huit réflecteurs, munis chacun d'une lampe de 150 watts, c'est-à-dire de 300 bougies, soit un total de 14400 bougies décimales ${ }^{4}$.
\end{abstract}

Les créateurs lumière qui travaillent sur la durée ont pu mesurer également cette augmentation des niveaux, notamment en cas de reprise de spectacles créés une dizaine d'années auparavant, ce qui est souvent le cas à l'opéra. Tel le créateur

\footnotetext{
2 Les liens vers des sources numériques d'extraits de captations vidéo aideront à se les représenter.

3 Je ne m'attarderais pas ici sur le sujet dont on retrouvera les détails dans le premier chapitre « Entre Luxe et lux : naissance du noir » de mon livre : Noir Lumière et théâtralité, Villeneuve d'Asq, Presses universitaires du Septentrion, 2016.
}

4 JOUVET, Louis in « L'homme, l'électricité, la vie », Revue des Arts et métiers graphiques, numéro spécial 1937. 
lumière Christophe Fore ${ }^{5}$ qui analyse cette évolution en se référant à ses expériences, notamment Hamlet de Ambroise Thomas qu'il a éclairé dans la mise en scène de Moshe Leiser \& Patrice Caurier d'abord à Genève en 1996, puis au Covent Garden et à Barcelone en 2001, enfin au MET à New York en 20116. Présent à toutes ces reprises, il dit avoir été surpris des niveaux originaux particulièrement bas qu'il avait créés quand il voulut les reporter lors de la première reprise à Londres. Tout en respectant la création originale, il dut s'adapter à l'évolution de la vision des spectateurs et à la dimension des théâtres, notamment au MET où la scène et surtout la salle sont bien plus grandes : l'intensité lumineuse décroît au carré de la distance, donc beaucoup plus vite que la distance, ce qui joue sur la vision pour les spectateurs placés au loin. Dans ce cas, Christophe Forey a dû faire évoluer la lumière en l'augmentant tout en respectant l'esprit de la création originale. Dans d'autres cas, il fut surpris par les initiatives prises par les personnels des salles d'accueil et ne reconnaissait plus ses lumières dont les intentions dramaturgiques se trouvaient perturbées par ce besoin de montée en puissance du niveau des lumières. On peut également citer l'exemple des multiples reprises des Nozze di Figaro de Strehler créées dans de sublimes ambiances de clair-obscur et qui se sont peu à peu retrouvées suréclairées au point que Strehler avait fait retirer son nom de la production. Les photos de deux époques sont en soi explicites :

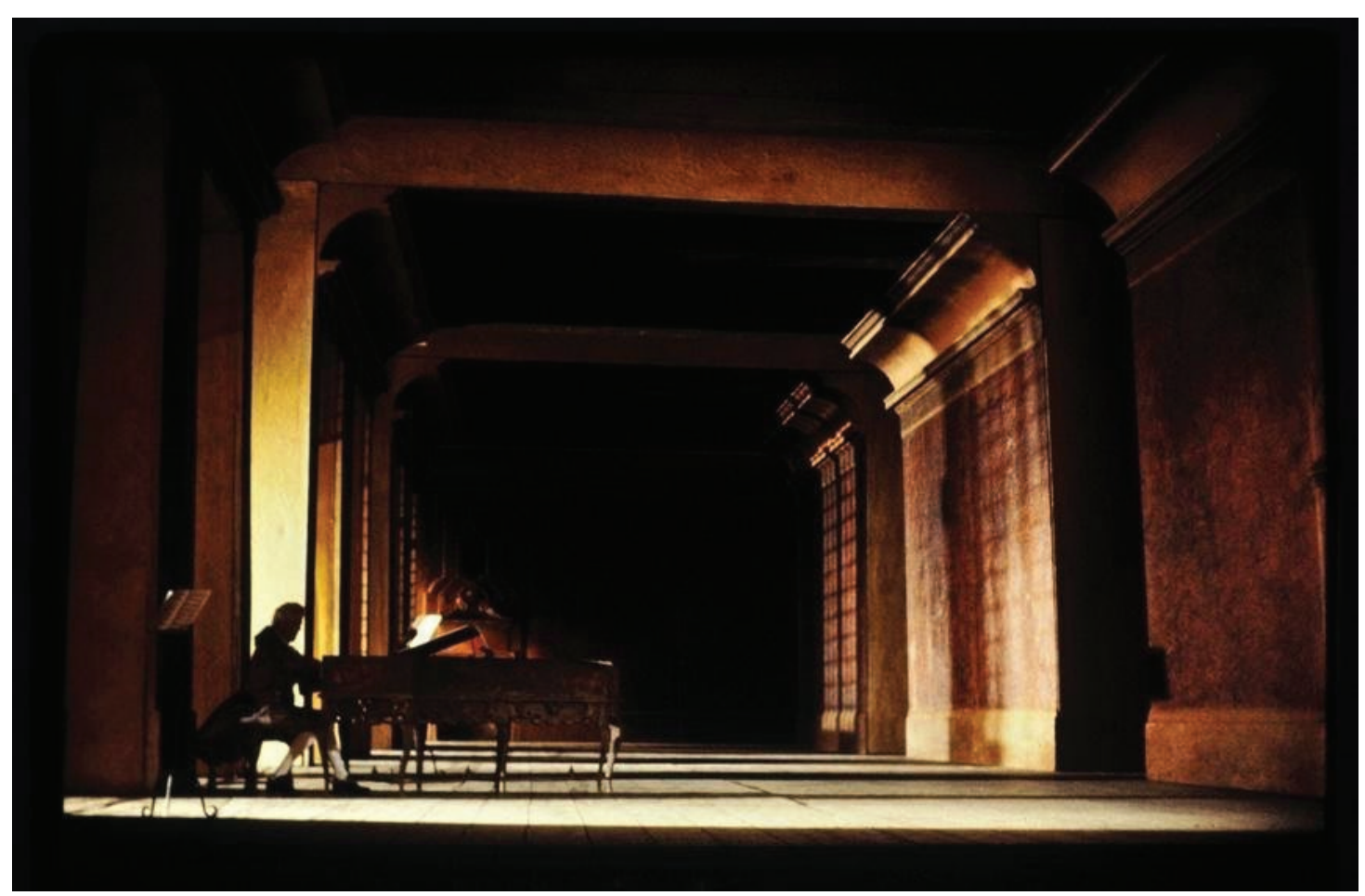

Les noces de Figaro, W. A. Mozart, mise en scène G. Strehler

Acte 3, photographie Daniel Cande,1980

(c) Bibliothèque Nationale de France

\footnotetext{
${ }^{5}$ Christophe Forey, créateur lumière de nationalité française, travaille à l'internationale pour l'opéra, la danse et le théâtre, notamment avec les metteurs en scène Moshe Leiser \& Patrice Caurier, la chorégraphe Lucinda Childs. Cf. http://www.christopheforey.org/lumiere/spectacles.html

${ }^{6}$ Témoignage recueilli lors de la préparation de la table ronde organisée par Antonio Palermo pour le colloque Tosc@ Paris, 27-29 juin 2019.
} 


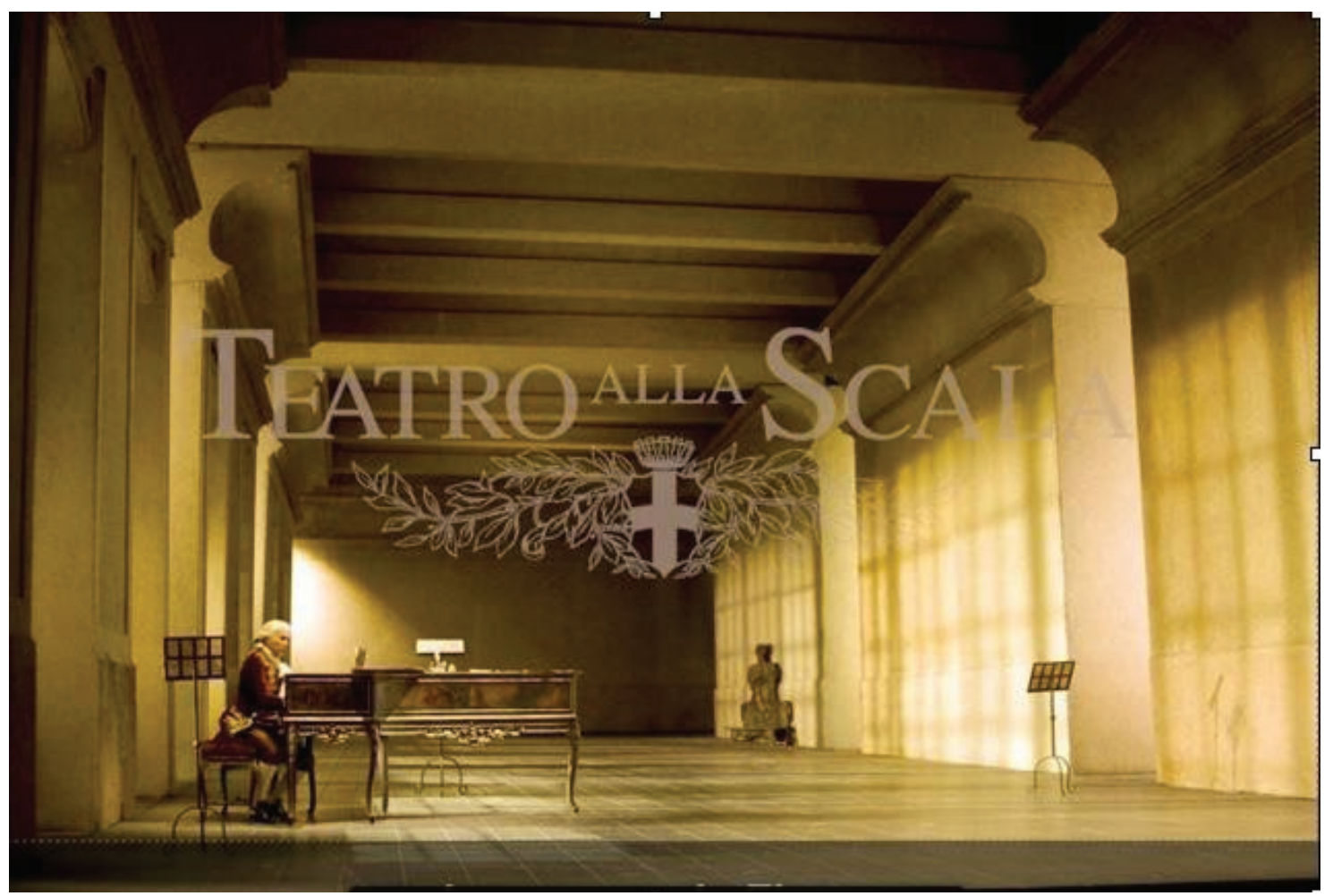

Les noces de Figaro, mise en scène G. Strehler (reprise) Acte 3, photographie Marco Brescia @ Teatro alla Scala, 2007

Christophe Forey en conclut que « la lumière est un élément vivant d'un spectacle, comme le jeu des acteurs ou des chanteurs, elle ne peut pas rester figée dans son premier geste ». Elle évolue donc avec les usages du monde. C'est un fait avéré que la lumière du quotidien a influencé la modification des niveaux de luminosité des spectacles. L'obscurité est devenue un luxe comme l'était inversément et historiquement la lumière jusqu'au XXe siècle. Le spectacle au XXe siècle c'est la lumière. D'autant plus que le protocole spectaculaire ritualise son lancement et sa clôture par un passage au noir. Mais au-delà, dans une histoire et un contexte culturels de la toute-puissance de la lumière, quelle est la place du noir?

\section{... à l'inconfort du noir}

Le noir par son histoire technique peut être perçu comme l'accident. L'extinction des flammes sortant des becs de gaz est un risque majeur au XIXe siècle. Outre l'absence de lumière, l'émanation de gaz toxique invisible qui s'ensuit génère une inquiétude bien réelle. Avec l'électricité, on craint la panne et la difficulté technique à la résoudre. Il est clair que ces risques sont actuellement obsolètes pour le gaz et sont prévus et anticipés concernant la panne électrique. Cependant, elles ont maintenu dans les mentalités l'association du noir à la crainte de l'accident et à la difficulté à se mouvoir dans l'obscurité. Les futuristes avaient joué en leur temps de cette peur, notamment dans un court événement scénique, Lumière de Francesco Cangiullo en 1922. Le principe était scénarisé en laissant les spectateurs dans le noir au lieu d'allumer la scène comme il se doit au bout d'un temps donné selon le protocole specta- 
culaire. Le scénario ${ }^{7}$ prévoyait une réaction orchestrée allant en s'amplifiant par des voix réclamant la lumière:

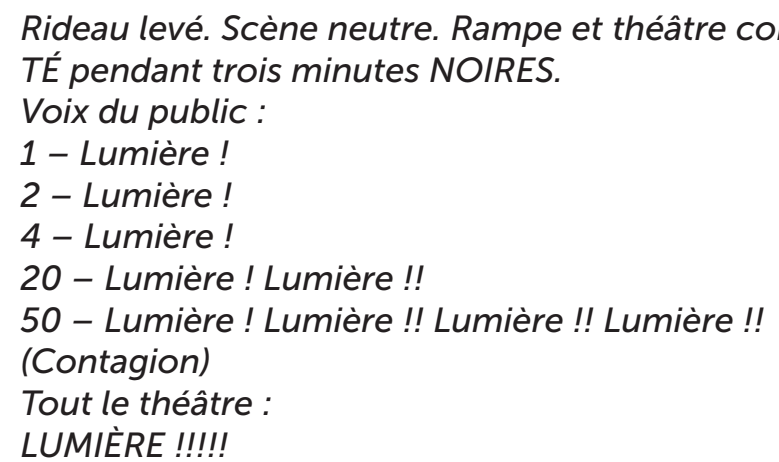

Il était prévu que ces appels se transforment en une crise « insensée ». Ensuite, une réponse non moins provocante consistait en une illumination de la rampe et du théâtre "EXAGEREMENT ». Ce spectacle futuriste illustre parfaitement cette situation qui devient non seulement son ressort dramatique jusqu'à l'exaspération, mais montre combien le spectateur en est tributaire : les spectateurs attendent une maîtrise de la lumière de la part des artistes. Dans le cas contraire ou par jeu, la situation vire au drame tant le noir est perçu comme un inconfort et un déni des attentes spectaculaires. Ce pouvoir peut s'avérer cynique lorsqu'on plonge ou maintient le spectateur dans un noir sans rien lui donner à la place.

Tant que l'on considère la lumière comme le principe de base, le noir (hors conventions protocolaires admises) peut être perçu comme une violence. Il peut être perçu et vécu comme rupture brutale non seulement parce qu'il aurait été amené sans ménagement par un cut, mais aussi parce qu'il incarnerait une rupture de confiance. Nos modes de vie nous mettent continuellement dans un environnement lumineux. Seuls les protocoles spectaculaires (cinéma et spectacle vivant) admettent le noir. Et encore très relativement puisque les signaux lumineux de sortie de secours sont toujours là pour nous rassurer. Le noir non protocolaire est potentiellement perçu comme une action violente et brutale. Ce que certains spectacles ont utilisé comme ressort dramatique (scénique) et dramaturgique (signifiant).

$C^{\prime}$ est le cas de Salves de Maguy Marin (2012) ${ }^{8}$. C'est un spectacle qu'on a peine à qualifier de chorégraphique si l'on se réfère à la tradition. C'est pourtant un spectacle qui repose sur le mouvement, le noir en étant le principal régulateur. Les situations commencent à s'installer puis sont systématiquement sapées dans leur élan par un noir brutal et net : une femme commence à recoller les morceaux d'un vase qui s'est brisé au sol, mais son geste est interrompu par un noir. Il ne reste au spectateur qu'à imaginer la suite, c'est-à-dire la remise en forme complète du vase brisé. Or, le noir rompt aussi le flux narratif : le mouvement d'ensemble se construit à rebours de la continuité. Lorsqu'une séquence ultérieure montre la même femme au vase en train d'y mettre des fleurs, on ne sait plus s'il s'agit d'un retour en arrière (ce vase est-il le

\footnotetext{
Voir LISTA, Giovani, Futurisme. Manifestes Documents Proclamations, L'Âge d'homme, 1973.

${ }^{8}$ https://www.lightzoomlumiere.fr/realisation/salves-cie-maguy-marin-un-choc-visuel/
} 
vase d'avant sa chute ?) ou une anticipation par saut elliptique. Le spectateur sans cesse arrêté dans le suivi des situations, est malmené par des noirs qui contrecarrent son effort mental de reconstruction. C'est un spectacle qui épuise littéralement le spectateur. Le noir gagnant sur le mental. Par salves, comme son titre, ce spectacle donne et reprend. C'est la victoire du noir sur le spectateur et le dramatique. Le fil conducteur, invisiblement tissé par les sept interprètes en ouverture, fait allusion à « la perte de l'expérience » dénoncée par Walter Benjamin'. Selon lui, la difficulté de transmettre aussi bien nos expériences privées que celles de l'humanité conduit à un appauvrissement qui menace les générations futures et fragilise le lien. Le mouvement de l'Histoire est rompu et la continuité perdue. Dans ce spectacle, d'un noir à l'autre, l'histoire de l'art conjointement à l'Histoire de l'humanité se brise : la statue de la liberté ou la Vénus de Milo tombent au sol comme autant de repères fragiles. Pour le spectateur, la perte de ses propres repères lui permet d'éprouver les catastrophes collectives du XXe siècle qui ont transformé le présent en un champ de ruines.

Bien qu'antérieur, le spectacle N d'Angelin Preljocaj créé en 2004 est, selon les propos du chorégraphe, un «titre masque ", tant ce que le spectacle évoque et fait éprouver est de l'ordre de l'innommable, de l'indicible : la souffrance, l'humiliation et la torture imposées au corps tendent à l'annihiler. C'est ce que dénonce N. Pour ce propos engagé, Angelin Preljocaj et ses collaborateurs Ulf Langheinrich et Kurt Hentschläger ont exposé les corps en scène à cette violence que relaie celle faite au spectateur dont les facultés sensorielles sont mises à mal ${ }^{10}$. Le son particulièrement fort essentiellement dans les infrabasses, associé à une très longue séquence qui utilisaient des stroboscopes très puissants (le spectacle était déconseillé aux épileptiques et aux femmes enceintes), crée un effet d'oppression et d'agression propice à la dénonciation. L'enjeu d'un tel spectacle est de montrer et de dénoncer en faisant ressentir. Les alternances de lumière et de noir sont instrumentalisées de manière consciente jusqu'à épuisement. Le spectateur piégé n'a pas d'échappatoire : les infrabasses résonnent dans son corps comme les jeux de lumière agressent ses yeux.

Dans ces spectacles non verbaux, le visuel prend une dimension primordiale. Le spectateur averti, bien qu'en état de choc répété, est généralement apte à admettre et à adhérer à cette nouvelle convention. Sa souffrance et son mal-être sont une nouvelle forme d'empathie participative à la violence dénoncée. Dans le cas du théâtre verbal, le contexte est tout autre. Le support du visuel pour accompagner le son est attendu, excepté lorsqu'il s'agit d'un enregistrement. Le son de la voix amplifié supporte le noir, tandis que le texte dit par un comédien sur scène sans amplification nécessite le raccord visuel à sa source. L'humain (l'animal ?) est habitué à l'alchimie du message oral associé aux indicateurs non verbaux, quand bien même il est actuellement rompu aux communications de toutes sortes à distances. Mais dans le protocole du face-à-face qui caractérise justement le spectacle vivant, il est dans cette attente.

\footnotetext{
9 BENJAMIN, Walter, «Expérience et pauvreté », in CEuvres, t. 2, Trad. Maurice de Gandillac, Painer Rochlitz et Pierre Rusch, Gallimard, 1972, 1974, 1977, 1978, $1985,1989,2000$, p. 364-372.

${ }^{10} \mathrm{https}: / /$ www.bing.com/videos/search?q=Angelin+Preljocaj++N\&pc=cosp\&ptag=G6C999N1234D010316A316A5D3C6E\&conlogo=CT3210127\&ru=\%2fsearch\%3fq\%3dAngelin\%2520Preljocaj\%2520\%2520N\%26pc\%3dcosp\%26ptag\%3dG6C999N1234D010316A316A5D3C6E\%26form\%3dCONBDF\%26conlogo\%3d $\underline{\mathrm{CT} 3210127 \& v i e w=d e t a i l \& m m s c n=v w r c \& m i d=034 B 96 F 66 B 4 D 4 C 7021 E C 034 B 96 F 66 B 4 D 4 C 7021 E C \& F O R M=W R V O R C}$
} 
Dans les années 1970-1980, le metteur en scène André Engel était connu dans son travail avec l'éclairagiste André Diot pour mettre le spectateur mal à l'aise en lui enlevant le confort de la visibilité du plein feu. Ils mettaient les spectateurs en situation de fournir un effort pour voir et entendre et non pas seulement consommer en regardant et en écoutant. Ils ne plongeaient pas les spectateurs dans le noir mais dosaient le niveau si bas, à l'unisson des voix des acteurs, que le spectateur était obligé de se mettre dans une tension parfois jusqu'à la rupture en quittant la salle. Le festival d'Avignon bien connu pour ses scènes de provocations, tant du côté des artistes que des spectateurs qui n'hésitent pas à manifester leur désapprobation, en fut le cadre lors de sa mise en scène de Venise sauvée de Hugo von Hofmannsthal en $1986^{11}$.

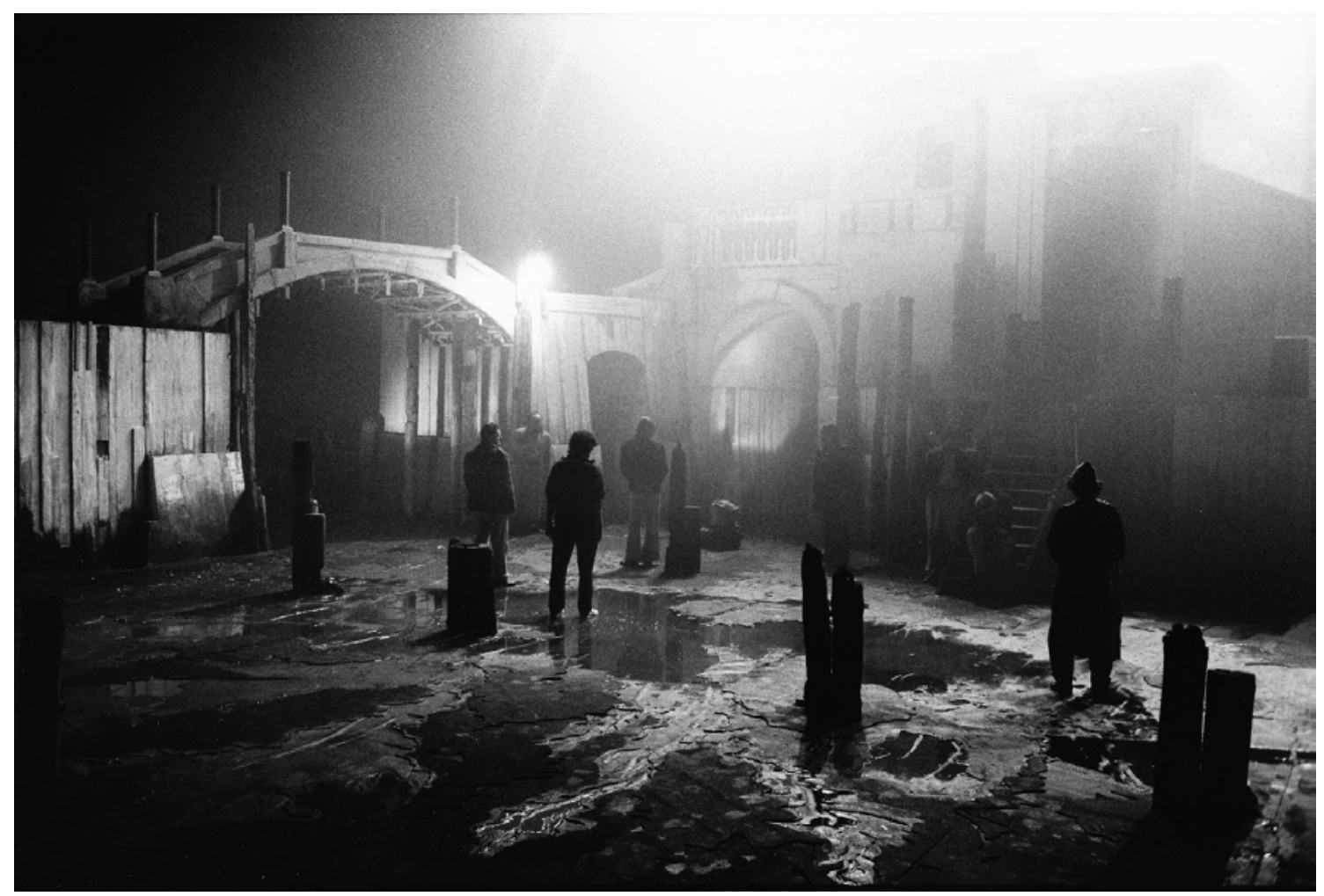

Venise sauvée Photo Brigitte Enguerand

Le décor conçu par Nicky Rieti d'un réalisme qui s'apparentait à celui d'un studio de tournage représentait la Venise des bas-fonds, insalubre, mais qui portait encore un peu de sa splendeur passée. "Un trou d'air, sans lumière, puant le poisson pourri », dit l'héroïne. Prenant le texte à la lettre, l'équipe artistique en fera une réalité, transformant le lieu anonyme du gymnase du lycée Aubanel d'Avignon en un port, celui d'une Venise lacustre le soir, sans lumière autre que celle, blafarde et partielle, de quelques lampadaires. Loin de « la verticalité des flammes », rappelée par Bachelard, celle des lampadaires urbains rabattait au sol toute aspiration à échapper au destin.

11 Voir PERRUCHON, Véronique, André Engel. CEuvre théâtrale, Villeneuve d'Ascq, Presses universitaires du Septentrion, 2018, pp. 122-129. 
Dans ce décor, ce qui comptait pour l'équipe artistique, c'était de créer l'atmosphère de conspiration sur fond de désespoir amoureux. Ainsi, la nuit fut épaissie par une brume permanente qui obscurcissait encore davantage la scène en barrant l'horizon. Le brouillard associé à une nuit obsédante couvrait toute la salle d'un voile épais dans une atmosphère pesante. Certes, il s'agissait d'une expérience polysensorielle pour le spectateur, mais cette atmosphère créait une tension difficilement supportable tant l'obscurité sur scène témoignait, comme le disait alors Georges Banu, d'un « appétit d'irreprésentable $\mathrm{e}^{12}$ ». André Diot ${ }^{13}$ avait conçu un éclairage non éclairant, innovant une forme d'esthétique scénique en pariant sur la capacité rétinienne du spectateur à s'adapter à l'obscurité. Loin de la convention, la nuit vraiment représentée fut un pari osé qui n'eut pas que des partisans. Car finalement dans cette démarche provocante, le noir de la nuit et cette lumière non éclairante n'avaient pas seulement pour finalité de rendre compte d'une réalité et d'une atmosphère de conspiration. Au-delà de l'esthétique et de la dramaturgie, il y avait de la révolte. Engel revendiquait de mettre le spectateur en état de tension. Ce fut le cas et le spectacle se finissait sous les huées ou les applaudissements, selon qu'on était sensible à l'esthétique et à la cohérence de l'ensemble ou selon qu'on se sentait frustré. Si le spectateur n'était pas personnellement visé dans cette tourmente, il n'empêche qu'il s'y perdait. Car l'effet du brouillard associé à celui de la nuit était accentué par un traitement sonore déroutant. Un texte souvent inaudible parce que chuchoté, bien qu'Anne Alvaro jouât avec les brisures de sa voix, rendant palpable la détresse de son personnage. Ce fut trop peu pour rendre intelligible le texte. Venise sauvée était un spectacle qui n'allait pas de soi et qu'il fallait aller chercher.

Pourtant, on a beaucoup parlé de la beauté de ce spectacle noyé dans la brume en faisant l'éloge des images. Si la flamme comble de plaisir le rêveur qui l'observe dans le noir à l'instar de Bachelard, la nuit obscure sans l'ombre d'une lueur à l'horizon désespère celui qui s'y confronte. Ce théâtre repose sur l'adhésion de partisans et non pas sur le confort de celui qui assiste à une représentation. C'est un théâtre engagé au-delà de ce qu'on attend du spectateur.

Ainsi en fut-il de ces types de spectacles qui reposent sur la discordance des attentes par une expérimentation de la violence du noir. Dans le noir, pour que l'expérience porte ses fruits et que le message passe, une relation de confiance est nécessaire.

\section{De l'apprivoisement du noir...}

Le noir se laisse apprivoiser de part et d'autre de la rampe lorsque la cohérence dramaturgique est au rendez-vous. Il faut se donner le temps et les moyens de s'y confronter physiquement. La responsabilité incombe aux créateurs qui verront d'un point de vue nouveau le noir. En dehors du pis-aller ou du protocole cadrant, le noir est un outil de construction dramatique du spectacle et une composante de la pa-

\footnotetext{
12 BANU, Georges, Art Press n 110, janvier 1987.

13 André Diot, créateur lumière né en 1935 a notamment été le collaborateur au théâtre et à l'opéra d'André Engel et de Patrice Chéreau pendant plus de 40 ans. Venu du cinéma, on lui doit en France l'apport des projecteurs HMl au théâtre.
} 
lette lumière. La Magie nouvelle (courant porté par la Compagnie 14:2014) en a fait un usage dramatique au service d'une construction dramaturgique qui implique le spectateur. Le noir est certes un protocole indispensable aux effets magiques, néanmoins, en tant qu'instrument dramatique au service d'une dramaturgie, il vise à faire entrer le spectateur dans un déséquilibre des sens qui mène à un détournement du réel. La magie repose toujours sur le principe de déstabilisation du spectateur. Ici, la compagnie vise l'adhésion à un réel modifié. Cela passe par un accompagnement qui relève d'un changement d'habitudes et une déconstruction pour le spectateur et l'acteur de ce que Marcel Mauss puis Pierre Bourdieu ont nommé l'habitus. Cependant, la mutation n'a que la durée d'un spectacle pour opérer. Aussi, le noir séquentiel joue un rôle fondamental dans cet accompagnement tout en ayant une fonction dramatique et dramaturgique. Cette alchimie maîtrisée par la Compagnie 14:20 fonctionne particulièrement bien dans des spectacles comme Vibrations (2010) ou Wade in the water (2016) ${ }^{15}$ qui raconte l'histoire d'un homme confronté aux effets d'une tumeur au cerveau qui lui font perdre ses repères au sens propre et figuré. Le spectateur voit le protagoniste (Aragorn Boulanger) en apesanteur, évoluer au ralenti ou avoir le don d'ubiquité. La gravité du sujet, les effets physiologiques de la maladie et les perturbations qui en découlent avec son entourage familial, se ressentent pour le spectateur d'autant plus intensément qu'il est plongé dans une atmosphère ténébreuse.

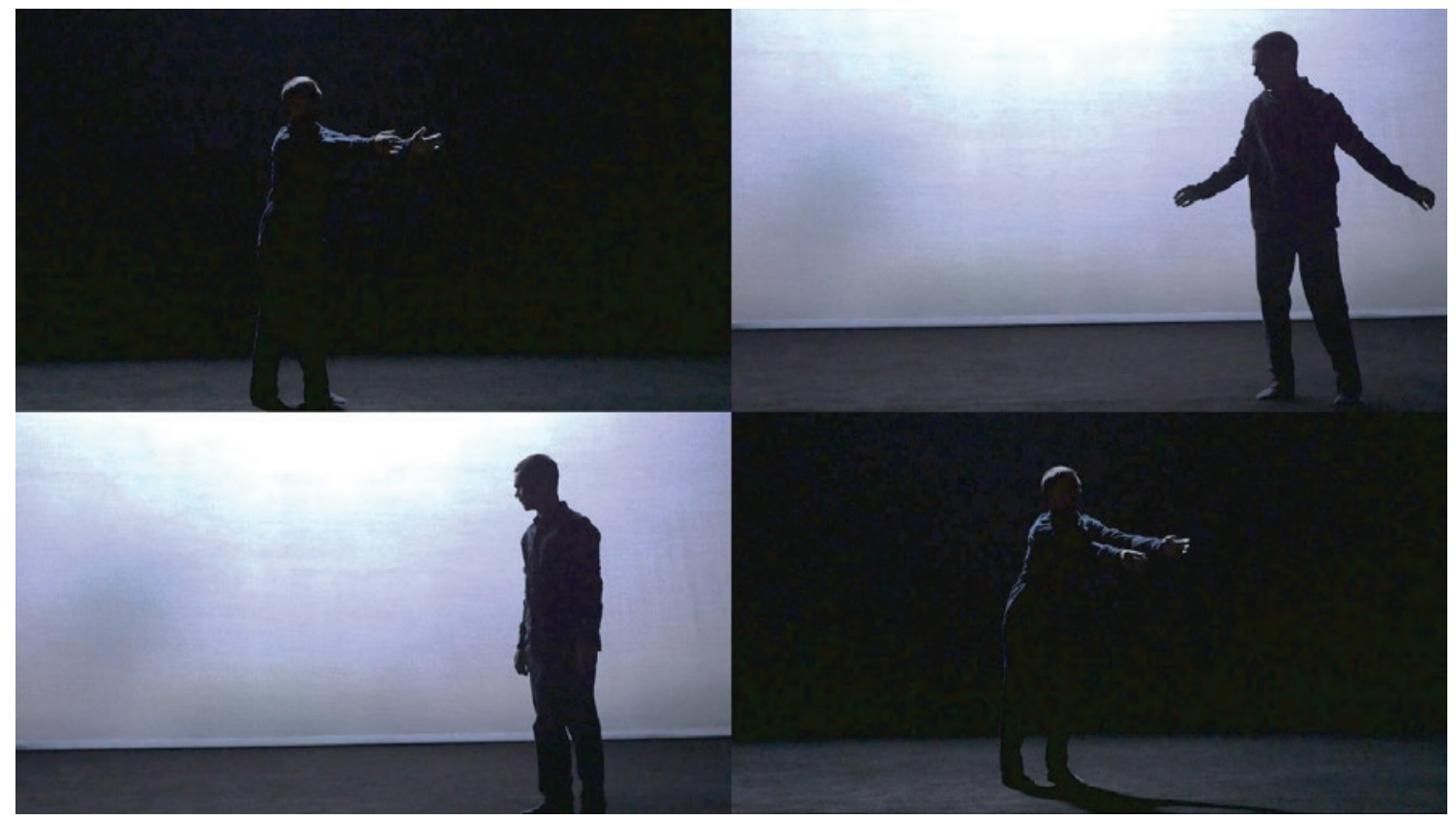

Wade in the water

Crédit photo : Cie 14:20

Les corps en scène y défient les lois de la gravitation et de la logique. Ils bous-

\footnotetext{
${ }^{14}$ La Magie nouvelle, courant impulsé et porté par la Cie de cirque 14:20, est un label créé par Raphaël Navarro, Clément Debailleul et Valentine Losseau, respectivement magicien, circacien et anthropologue, lors de la création de la Cie en 2000.

${ }^{15}$ Wade in the water, création 2016, conception et mise en scène : Clément Debailleul et Raphaël Navarro, chorégraphie : Aragorn Boulanger, lumière : Elsa Revol. https://cie1420.jimdo.com/cr\%C3\%A9ation/spectacles/wade-in-the-water-1/
} 
culent la rationalité de leur présence et de leur absence ou de leur démultiplication dans un réel recréé auquel le spectateur s'associe grâce aux noirs qui rythment le spectacle. Intégré à la dramaturgie et à la poétique scénique dès sa gestation, le noir n'est pas perçu artificiellement comme une rupture brutale qui violenterait le protocole en cours. Le processus d'acheminement du spectateur est contenu dans la dramaturgie même du spectacle. De moyen, le noir devient une composante du spectacle. Dans Wade in the water, les noirs instaurent un rythme qui permet de déjouer celui du spectacle. C'est-à-dire que par un phénomène de répétition, le spectateur inscrit des attentes qui lui sont connues - on fait le noir pour changer le décor, installer une ellipse temporelle, marquer un changement de scène, etc. Ce sont les noirs du réel et du rythme d'un spectacle. C'est rationnel. Mais au fur et à mesure, cette réalité est amenée à disparaître au profit d'une autre à laquelle le spectateur est arrivé grâce à ces paliers, sans s'en rendre compte. Un univers où les corps flottent, s'envolent et perdent de leur consistance. Réalité nouvelle qu'il intègre de ce fait sans difficulté. C'est en jouant des perceptions du spectateur, en éveillant l'esprit à de nouvelles expériences sensorielles par association et en modifiant les repères culturels, cognitifs et sensoriels du spectateur que la magie se manifeste.

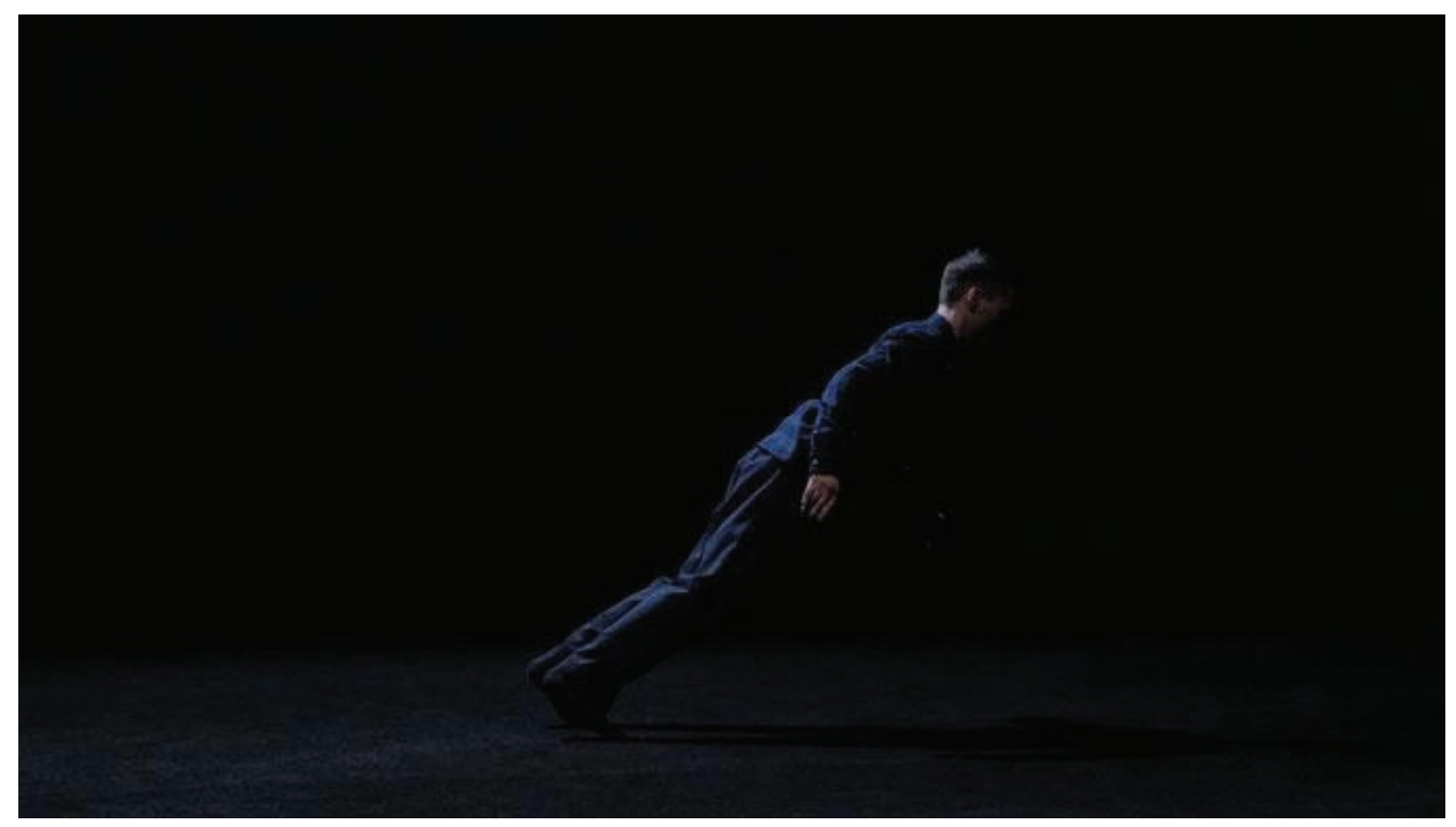

Wade in the water [Wade na água]. Crédito da foto: Cie 14:20.

Cela est vrai pour le spectateur qui va adopter le noir comme une composante et pour le créateur qui va le maîtriser pour apprivoiser le spectateur sans l'effrayer afin de le conduire dans un réel modifié. Mais qu'en est-il pour le performeur?

Aragorn Boulanger qui travaille avec la lumière d'Elsa Revol construit cette relation au noir notamment dans Ellipses (2012) ${ }^{16}$. Dans ce spectacle, Aragorn Boulanger

${ }_{16}$ https://www.bing.com/videos/search?q=Ellipses+Aragorn+Boulanger\&pc=cosp\&ptag=G6C999N1234D010316A316A5D3C6E\&conlogo=CT3210127\&ru=\%2fsearch\%3fq\%3dEllipses \%2520Aragorn\%2520Boulanger\%26pc\%3dcosp\%26ptag\%3dG6C999N1234D010316A316A5D3C6E\%26form\%3dCONBDF\%26conlogo\%3dCT3210127\&view=detail\&mmscn=vwrc\&mid=18B66C52192F645B6B2B18B66C52192F645B6B2B\&FORM=WR$\underline{\text { VORC }}$ 
décline la question des rythmes du corps en interaction avec la lumière et les noirs : le corps est comme arraché aux lois de la physique qui sont troublées dans un temps et un espace redessinés par les lumières d'Elsa Revol dont le noir est une composante. Concrètement, il dessine un geste scénique qui est interrompu par un noir, puis qui reprend lors du rallumage, non pas là où on l'avait laissé, mais là où l'a mené son prolongement dans le noir. Le personnage nous emmène au seuil de la réalité et du rêve, car ses gestes lents, souvent répétitifs, finissent par embrouiller les repères que le cerveau se donne dans cette reconstruction mentale qui finalement lui échappe. Il semble chercher un geste, une place dans l'espace de la scène. Pour que chacun de ses gestes devienne la métaphore d'une ultime traversée, Aragorn Boulanger travaille sur un état de dissolution dont l'expérience s'imprime corporellement en lien avec la lumière ou le noir. Dans Ellipses, l'allumage, l'extinction, l'intensité ou la direction de la lumière sont pilotés au cours même de la performance qui transfigure l'espace et offre une vision différente au spectateur du geste chorégraphié. Le noir dans ce cas est tout sauf un noir. Il n'est pas un obstacle mais permet au contraire de créer un espace où les repères réels et irréels sont floutés ou même inutiles : seul son propre corps est un repère pour tout mouvement, déplacement, ralentis, accélérations. Le noir induit un changement d'être qui passe par une exacerbation de la proprioception et apporte cette qualité de rapport corporel à soi qu'expérimentent ceux qui jouent dans le noir alors même qu'ils sont voyants. Tout comme la lumière, le noir est ici un support plastique et environnemental, mais aussi un moyen de donner au corps disparu, mais deviné, un poids et une dynamique dédouanés de la gravitation et de la masse de sa corporalité. Le noir participe à l'écriture propre aux reconstitutions cognitives et au travail du cerveau, entre visibilité et invisibilité, phénomènes d'apparitions et de disparitions inattendues.

\section{... à la communion profonde}

Le cas de la Magie nouvelle est particulièrement fécond pour repenser le lien au noir parmi les composantes scéniques. Dans cette forme spectaculaire, le pacte du spectateur ne repose plus sur le critère de connivence dans le cautionnement de l'effet magique (ce qui est le cas dans la magie traditionnelle), mais sur l'acceptation du détournement du réel. La Magie nouvelle installe progressivement de nouveaux repères cognitifs auxquels le cerveau adhère acceptant que, dans ce monde, les corps flottent, se meuvent au ralenti ou extrêmement rapidement, disparaissent et réapparaissent, se démultiplient, etc. Processus dans lequel le noir joue une place subtile fondamentale. Ainsi, le temps d'un spectacle, l'adhésion commune aux acteurs et aux spectateurs autour d'une réalité modifiée ou détournée, donc d'une nouvelle réalité, relève de celle que partage toute communauté à travers sa propre culture ${ }^{17}$. L'expérience de la Magie nouvelle offre la possibilité de "sortir de sa zone de confort ${ }^{18}$ » selon l'expression reprise par les psychologues du comportement, si tant est que cette zone existe réellement. Dans ce contexte singulier, spectateurs, créateurs et interprètes apprennent à penser la scène avec le noir. En prenant appui sur la pra-

\footnotetext{
17 Ce que Valentine Losseau anthropologue et dramaturge de la cie 14:20 étudie à travers les préjugés cognitifs, https://www.artefake.fr/valentine-losseau/

18 Expression dont l'origine prend sa source dans les études de R. Yerkes \& J. Dodson, "La Danse de la Souris, Une Étude du Comportement Animal", 1907.
} 
tique et les particularités de la Magie nouvelle qui invitent à bousculer les repères, il s'agirait, grâce au noir scénique, d'éprouver la possibilité de couper les liens qui nous enferment dans une représentation dominée par la peur et le rejet de l'obscurité. En un mot, il s'agirait de se " déterritorialiser » (Gilles Deleuze). Le noir articulé à la dramaturgie et à la dimension dramatique de la scène dans les spectacles de la Magie nouvelle cités plus haut, nous offre l'opportunité de repenser sa présence scénique et par là même, ses fonctions et son efficacité pour nous inviter à éprouver une liberté esthético-politique. En adhérant à un réel modifié nous faisons cette expérience. En nous confrontant au noir dans une fusion ou même une communion profonde, nous faisons cette expérience.

En définitive, de la dissonance du noir brut à la concordance du noir apprivoisé, au-delà et par le concret de la scène, le chemin parcouru nous mène à la pensée du monde. Car continuer à supporter ou accepter le noir comme un diktat de la peur, revient à perpétuer un processus mortifère pour la conscience humaine et l'art. Ce qui nous amène à avancer à l'instar de Félix Guattari que « le rapport de la subjectivité avec son extériorité - qu'elle soit sociale, animale, végétale, cosmique - se trouve ainsi compromis dans une sorte de mouvement général d'implosion et d'infantilisation régressive ${ }^{19}$ » dû à une standardisation culturelle. Concernant le rapport du sujet (artiste ou spectateur) à l'environnement du spectacle, lorsque le noir scénique est maintenu dans son rôle brutal, cela revient à une infantilisation du sujet et à l'implosion du bon déroulement du spectacle (comme dans la proposition provocante des futuristes). A contrario, quand la violence du noir est détournée au service d'une dénonciation comme le montrent les spectacles cités dans la première partie (Salves, $N)$, alors le noir va dans le sens d'une remise en question de son statut par amplification. La question reste de savoir si ces mises à l'épreuve ne représenteraient pas un risque de détruire l'agencement de subjectivation que le principe même du spectacle vivant propose. En un mot, n'y a-t-il pas risque de rupture, de fuite, et donc du retour de la peur sous-jacente qui détruit l'équilibre et impose sa loi ? Le dosage est fin entre la rupture et le dissensus consenti. Mais par un dosage et une approche du noir réussis, il est possible de dépasser les principes et les peurs pour plonger au plus profond du noir et finalement comme Baudelaire « Plonger au fond du gouffre, Enfer ou Ciel, qu'importe ? / Au fond de l'Inconnu pour trouver du nouveau !20».

Le noir apprivoisé et re-pensé permet une approche philosophique du monde perçu dans son entièreté où les sujets ne sont ni isolés de l'environnement, ni dominants, dans un dépassement de l'anthropocentrisme. Et ce, à l'instar de la philosophie de Arne Næss qui propose une pensée du monde selon une écosophie entendue comme philosophie d'harmonie ou d'équilibre entre les subjectivités quelles qu'elles soient. De fait, le noir, nous l'avons vu dans la deuxième partie, parce qu'il est un des sujets qui souffrent d'une pensée close se doit d'être re-considéré pour devenir potentiellement une composante forte d'une pensée holistique et écosophique du spectacle dans un monde en mutation. Grâce à l'expérience du noir, à l'unification et

\footnotetext{
19 GUATTARI, Félix, Les trois écologies, Paris, éditions Galilée, 1989, p. 12.

20 Dans l'édition de 1861, le dernier chapitre des Fleurs du Mal intitulé « La mort » comporte six poèmes. « Le Voyage » en est le poème final et les vers cités sont les deux derniers du poème et donc du recueil.
} 
à la perte des repères qu'il propose, cette pensée du rapport au monde peut s'adapter à l'univers du spectacle.

En définitive et en opérant un glissement d'une pensée écosophique du monde à une pensée du spectacle vivant comme micro-monde, cette réflexion a pour visée de proposer le noir comme vecteur potentiel de subjectivation et de singularisation pour le spectacle vivant. Autrement dit, du « foyer existentiel partiel ${ }^{21}$ » que serait le spectacle au sein du monde. Le noir au théâtre peut participer à l'articulation éthico-politique - que Guattari nomme également « écosophie ${ }^{22}$ » - entre les trois registres écologiques : l'environnement, les rapports sociaux et la subjectivité humaine. Dans ce cadre de pensée, le noir n'est plus un brutal et violent arrachement au confort de la lumière, mais un révélateur du dépassement de la suprématie de l'homme sur le monde. Le noir en composante de la lumière dans l'environnement scénique n'est plus seulement « machinique » et technique mais une " composante de subjectivation $^{23}{ }^{2}$. Apprivoisé, il devient partenaire du rapport au monde qu'incarne le spectacle. Loin de la conception debordienne, le spectacle n'est plus objectivé, mais vécu dans une articulation intersubjective sensible au sein d'une réflexion éthico-politique. Non plus fuir et craindre le noir, non plus le contraindre à une régulation protocolaire et dominante du spectacle, mais créer une relation de confiance avec un noir apprivoisé, compris, pénétré, pour un nouvel art de la scène.

Ainsi, le noir entendu comme un environnement puissamment enveloppant peut, dans la douceur de son accueil, accompagner la communion profonde que l'art scénique invite à vivre. De l'objectivation scénique à la fusion profonde, il invite à l'expérience ultime de la poésie comme nous le suggère le poète Junichiro Tanizaki pour qui le noir « suscite des résonances inexprimables ${ }^{24}$ » que seul le corps attentif et la conscience éveillée sauront déceler. Alors, la peur du noir fossilisé dans sa représentation sera dépassée. On saura trouver la beauté de sa profondeur poétique mise en résonance avec une « écologie profonde ${ }^{25}$ » qui repose sur une indistinction métaphysique entre les êtres humains et la nature. Le noir apprivoisé à travers une expérience de la profondeur et de l'intime peut engager une voie nouvelle de « rejet de l'image de l'homme-au-sein-de-l'environnement en faveur de l'image relationnelle du champ de vue total ${ }^{26}{ }^{\prime}$. Artistes, interprètes, spectateur, chercheurs et penseurs, à nous d'en raconter la nouvelle histoire dans une écosophie de l'art scénique à construire.

Reçu : $17 / 03 / 2020$

Approuvé : $17 / 03 / 2020$

\footnotetext{
21 GUATTARI, Félix, op.cit., p. 37.

22 Idem, p. 12

${ }^{23}$ Idem, p. 22

24 TANIZAKI, Junichirô, Éloge de l'ombre, trad. SIEFFERT, René, Éditions Verdier 2011, p. 37.

${ }^{25}$ Expression de Arne N/ESS, pionnier de l'échosophie, « deep ecology » est une thèse présentée pour la première fois en 1973 dans un article « The Shallow and the Deep Long Range Ecology Movement », traduit en français dans H.-S. Afeissa (ed.), Ethique de l'environnement. Nature, valeur, respect, Paris, Vrin, 2007.

${ }^{26}$ N/ESS, Arne, «Le mouvement d'écologie superficielle et le mouvement d'écologie profonde de longue portée » dans Une écosophie pour la vie, Paris, Editions Seuil, traduit du norvégien par Naid Mubalech, et de l'anglais par Pierre Madelin, sous la direction scientifique de Hicham-Stéphane Afeissa et Mathilde Ramadier, p. 120.
} 